\title{
Correction to: Modelling of Soil Behaviour with Hypoplasticity
}

\section{Correction to:}

D. Mašín, Modelling of Soil Behaviour with Hypoplasticity, Springer Series in Geomechanics and Geoengineering, https://doi.org/10.1007/978-3-030-03976-9

In the original version of the book, the following corrections have been incorporated:

Equation 5.1 has been updated in Chapter 5 .

Equation 7.14 has been updated in Chapter 7.

The correction book has been updated with the changes. 